\title{
3D-Kultur
}

\section{(Teil-)Automatisierung der liquid-overlay-Sphäroidkultivierung}

ALEXANDER KRÜGER, MIRJAM INGARGIOLA, MELANIE HÜTHER, LEONI A. KUNZ-SCHUGHART

ONCORAY, NATIONALES ZENTRUM FÜR STRAHLENFORSCHUNG IN DER ONKOLOGIE, MEDIZINISCHE FAKULTÄT UND UNIVERSITÄTSKLINIKUM CARL GUSTAV CARUS, TU DRESDEN UND HELMHOLTZ-ZENTRUM DRESDEN-ROSSENDORF

3D tumor spheroid models are increasingly applied in secondary, cellbased drug testing due to certain therapy-relevant in vivo-like characteristics. Automation of routine and delicate steps in spheroid culturing and processing, including the gentle well-to-well transfer of spheroids, facilitates spheroid-based assay standardization. Semi-automated workflows minimize experimental variations and are recommended in particular for combination treatment testing and long-term monitoring.

DOI: $10.1007 / \mathrm{s} 12268-016-0716-\mathrm{X}$

(C) Springer-Verlag 2016
Tumorsphäroide sind seit über 40 Jahren in der onkologischen Forschung im Einsatz und ein attraktives Modell mit intermediärer Komplexität für die Therapietestung. Ihre 3D-Architektur ist assoziiert mit der Entwicklung von Nährstoff- und Proliferationsgradienten, welche die Wachstumskinetik beeinflussen und das pathophysiologische Mikromilieu in Mikrometastasen bzw. Mikroarealen größerer Malignome in vivo widerspiegeln [1-4]. Im Wirkstoffscreening wird nach wie vor meist auf konventionelle 2D-Assays zurückgegriffen, obwohl bereits Sphäroide etablierter Tumorzelllinien eine bessere Selektion vielversprechender Wirkstoffe vor der Applikation

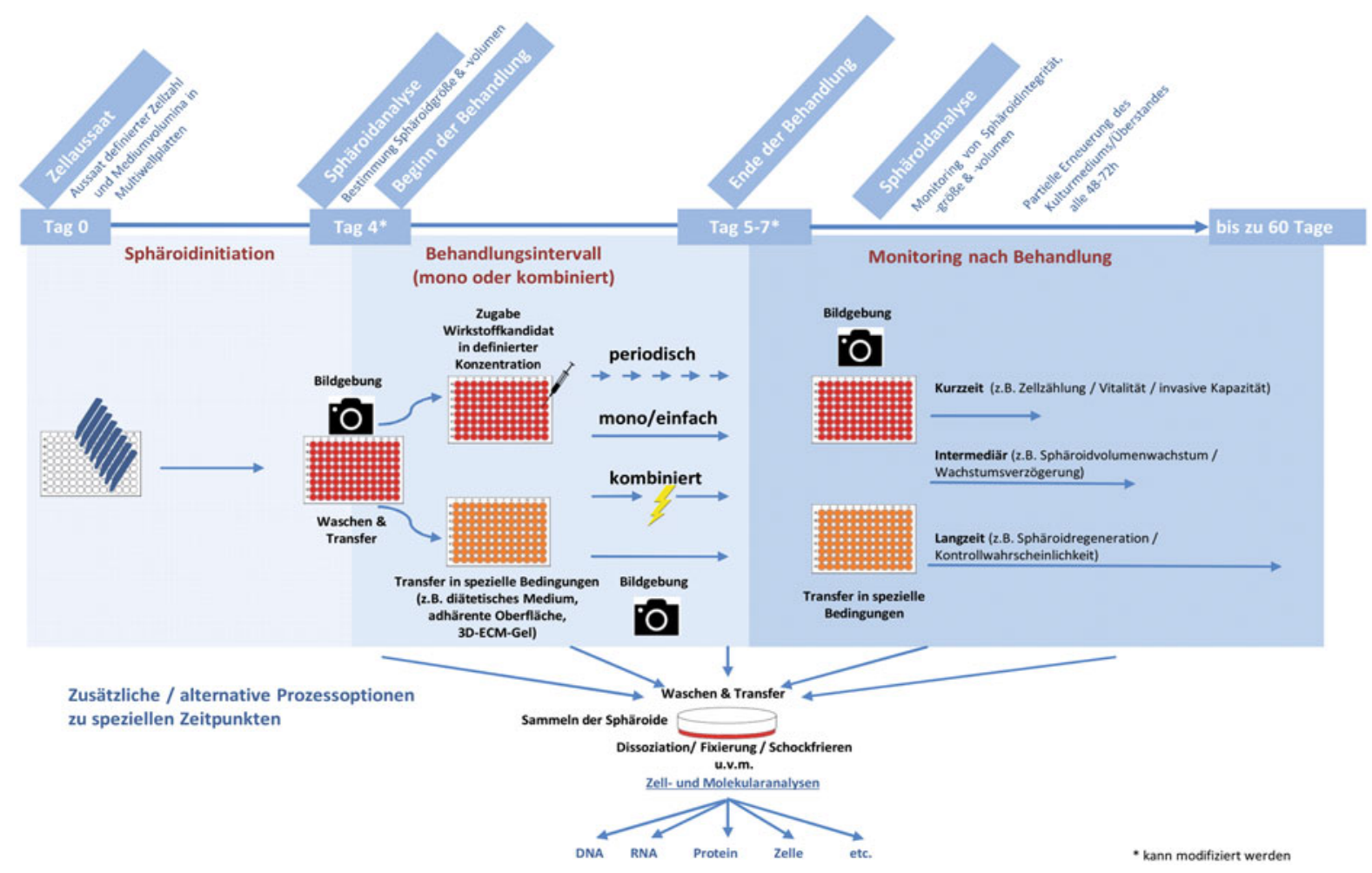

$\Delta$ Abb. 1: Schema zur (teil-)automatisierten Aussaat, Fütterung und Prozessierung von Sphäroidkulturen in der Therapietestung (modifiziert nach [10]). Nach der Initiationsphase erfolgt die Qualitätskontrolle der Sphäroidbildung mittels Phasenkontrastbildgebung. Im Behandlungsintervall sind z. B. Substanzzugabe oder Sphäroidtransfer in definierte Bedingungen automatisiert (Abb. 2). Abhängig von Fragestellung und Endpunkt sind regelmäßiges Füttern und Monitoring der Sphäroide hinsichtlich Integrität und Wachstum über wenige bis zu 60 Tage erforderlich. Langzeitkulturen über 30 Tage sind relevant für die Evaluation analytischer Endpunkte putativ kurativer Kombinationstherapien (z. B. SCD $_{50}=$ Sphäroidkontrolldosis 50 \%). 
im Tier erlauben. Die Nutzung des Modells setzt sorgfältig aus tumorbiologischen Erwägungen heraus definierte und kontrollierte Testbedingungen voraus. Kleine Sphäroide beispielsweise weisen 3D-Zellinteraktionen auf, jedoch nicht die oben genannten therapeutisch relevanten pathophysiologischen Charakteristika. Die Verwendung größerer Sphäroide in den für die Therapietestung bevorzugten Multiwell-Formaten setzt standardisierte Fütterungsmodalitäten zur Vermeidung zelltoxischer, pathophysiologischer Artefakte voraus [4-6].

Am Standort Dresden wird häufig, jedoch nicht exklusiv, der 96-Well-liquid-overlayAnsatz für die Therapietestung herangezogen $[5,7]$. Das Sphäroidvolumen zu Therapiebeginn variiert je nach Fragestellung; Standardgrößenklassen sind vor allem 350 bis 400 Mikrometer (ohne zentrale Sekundärnekrose) und 600 bis 700 Mikrometer (mit Sekundärnekrose und stark ausgeprägten Gradienten). In unabhängigen Studien konnten kürzlich über solche Größen-definierten
Sphäroidassays beispielsweise Substanzen identifiziert werden, die über eine Modifikation der mitochondrialen Atmung das Tumorzellüberleben reduzieren, vor allem in initial sauerstoffarmen (hypoxischen) Arealen - ein Phänomen des Tumormikromilieus, das in 2D-Kultur nicht reflektiert wird [8,9].

\section{Analytische Endpunkte}

Sphäroide können mit allen modernen molekularanalytischen Methoden kombiniert werden [1-4]. Die Präparation der Proben ist zu jedem Zeitpunkt der Kultivierung möglich (Abb. 1). Molekularanalytische Endpunkte wie Genexpressionsmuster sind jedoch nicht ausreichend, um die Effizienz neuer Wirkstoffe und Behandlungsstrategien zu beurteilen. Koloniebildung und Zellzahlbestimmung sind keine Option für eine standardisierte Therapietestung an 3D-Sphäroiden, da sie die Dissoziation in Einzelzellen erfordern. Kommerzielle 2D-Überlebensassays sind oft nicht 3D-kompatibel. Einer der wenigen validierten, dissoziationsfreien und hochdurch- satzfähigen Ansätze zur Quantifizierung des Zellüberlebens in Sphäroidkultur nach Chemotherapie basiert auf dem APH(acid phosphatase)-Assay [5, 7]. Sphäroidintegrität und -größe dagegen können einfach, schnell und wiederholt an vielen individuellen Proben mittels Phasenkontrastbildgebung dokumentiert werden. Ein entsprechendes Routinemonitoring in einer liquid-overlay-Langzeitkultur ermöglicht die Evaluation kurativer Therapieansätze, z. B. kombinierter Radiochemotherapien. Als analytische Endpunkte werden hierzu die Sphäroidwachstumsverzögerung sowie der prozentuale Anteil an Sphäroiden mit regenerativem Wachstumspotenzial dokumentiert [9-11]. Dies erlaubt die Ermittlung der Sphäroidkontrolldosis 50 Prozent, welche dem klinisch-relevanten Endpunkt einer 50-prozentigen Heilungsrate (Kontrolle) in Tumor-tragenden Tieren nahekommt. Solche Untersuchungen erfordern sehr lange Nachbeobachtungszeiten und viele Tiere bzw. Sphäroide pro Dosisgruppe. 
Teilautomatisierung der Sphäroidkultivierung und -prozessierung im liquid overlay

Die Automatisierung im Bereich 3D-Kultur dient nicht nur dem Zweck der Massenproduktion, sondern vielmehr der Standardisierung (Abb. 1). Durch automatisierte Zellaussaat kann zeitgenau eine große Anzahl an Sphäroiden mit minimaler Abweichung in Größe und Beschaffenheit zur Verfügung gestellt werden. In Agarose-beschichteten 96-Well-Platten sind Durchmesser mit intraexperimentellen Abweichungen von zwei bis fünf Prozent (zelltypabhängig) zu gewährleisten bei einer Aussaat von bis zu 20 Platten aus einer Zellsuspension. Eine ähnliche Qualität wurde 2009 für zwei kolorektale Sphäroidmodelle mit einer inzwischen nicht mehr erhältlichen Pipettiereinheit erreicht [10] und kann auch durch eine gut eingearbeitete Fachkraft erzielt werden. Bei der Bestückung höherer Plattenzahlen sind Reanalyse oder Ersatz der Zellsuspension angeraten.

Vor der Aussaat erfolgt manuell die Präparation definierter Einzelzellsuspensionen aus Monolayerkulturen. Die interexperimentelle Variation wird durch deren Herstellung und Qualität mit beeinflusst. Ein Vergleich der Sphäroidgrößen an Tag 4 in Kultur von zwei HNSCC(head and neck squamous cell carcinoma)-Sphäroidmodellen bei unabhängiger Vor- bzw. Nachbearbeitung durch fünf Experimentatoren, aber automatisierter Aussaat ergab Abweichungen von unter zehn Prozent. Gegenwärtig nutzen wir ein Biomek4000-System der Firma Beckman Coulter mit AchtKanal-Pipettieraufsatz, das neben Flüssigkeiten und Einzelzellsuspensionen auch intakte Sphäroide pipettiert. Zur Zellaussaat hat sich folgende Vorgehensweise bewährt: (1) Befüllen eines Vorratsbehälters mit Zellsuspension definierter Konzentration; (2) Aspiration unter Verwendung von 1.000Mikroliter-Spitzen und Mehrfachabgabe von je 200 Mikrolitern Zellsuspension pro Well mit Restvolumen (Aussaat von 40 Wells in einem Pipettierschritt); (3) programmierter Mixschritt vor jeder Probenaufnahme zur Gewährleistung einer homogenen Zellsuspension. Danach werden die Kulturplatten vibrationsarm und störungsfrei mehrere Tage standardisiert im Brutschrank inkubiert (= Initiationsphase).

Da Sphäroide nicht an der Kulturoberfläche anhaften, kann der Überstand bei Wirkstoffbehandlung und Routinefütterung nicht vollständig ohne Verlust oder Beschädigung

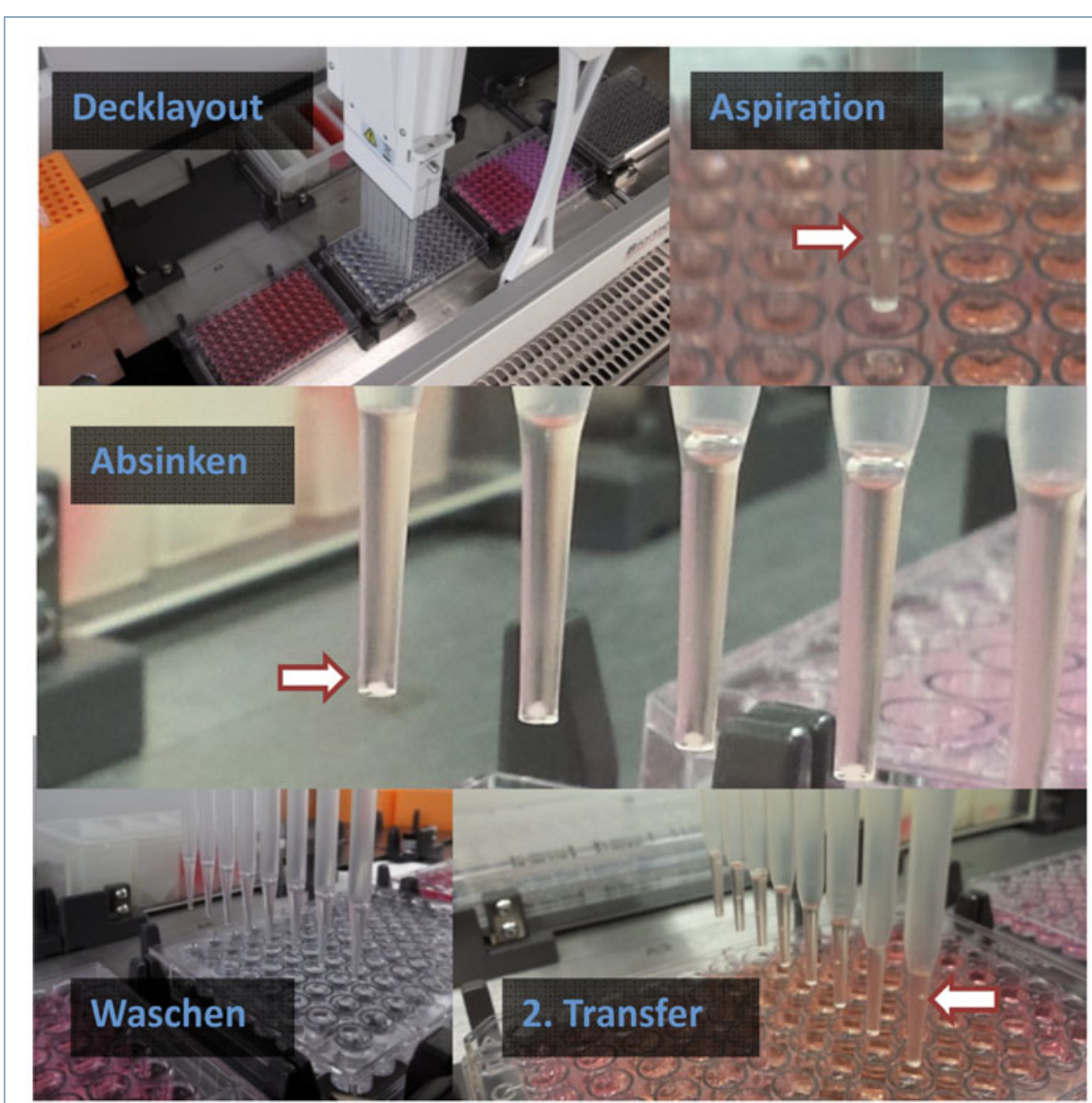

Automatisierter Prozess: Sphäroidtransfer \& Wirkstoffverdünnung

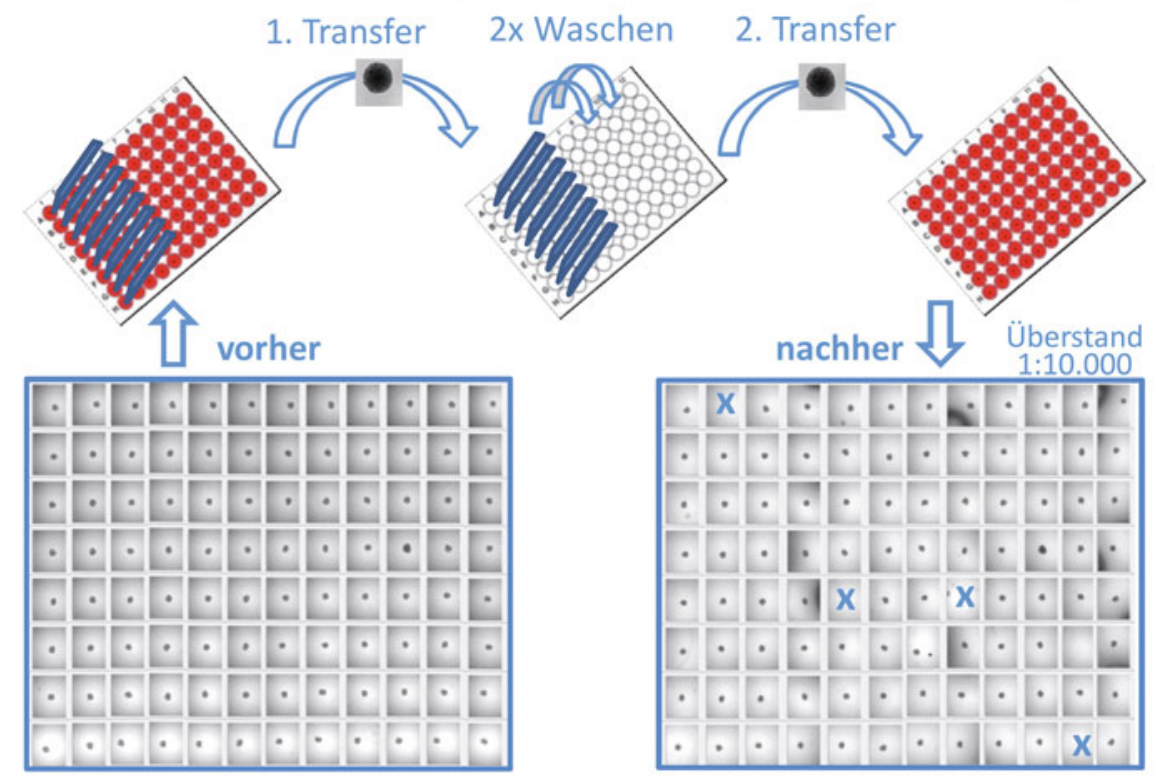

$\Delta$ Abb. 2: Sphäroidtransfer und Wirkstoffverdünnung mittels automatisiertem Pipettierschema. Oben fotografisch dokumentiert: Decklayout und erfolgreicher Ablauf der Methodik mit Sphäroidaspiration, Absinken in der Spitze, Verdünnungsschritt und Transfer. Unten ist die hohe Transfereffizienz exemplarisch für eine 96-Well-Platte bei einem Gesamtdurchlauf des automatisierten Protokolls zur Wirkstoffverdünnung $(1: 10.000)$ gezeigt.

der Kulturen abgesaugt werden. Bei manueller Handhabung wird üblicherweise ein defi- niertes Volumen abgenommen und ersetzt. Kritisch hierbei ist, dass Medium während 
der Inkubation vor allem in den Randbereichen der Multiwellplatten verdampfen kann - auch unter Wasserdampfsättigung. Dies ist in unterschiedlichen Wells, Platten und Inkubatoren verschieden stark ausgeprägt und im manuellen Set-up nicht ausgleichbar. Der methodisch-technische bias führt im Falle einer Therapieevaluation zu erhöhten Abweichungen, auch wenn die äußeren Wells analytisch ausgeschlossen werden. Dies lässt sich mittels speziell programmierbarem Pipettiersystem deutlich reduzieren. Wir entwickelten hierzu ein automatisiertes Pipettierschema am Biomek4000, bei dem durch definierte Eintauchtiefe der Pipettenspitzen genau 50 Prozent des Ausgangsvolumens mit Sphäroid im beschichteten Well verbleiben (100 Mikroliter). Der Wirkstoff bzw. frisches Medium kann somit in exaktem Volumenverhältnis zugesetzt werden.

Ebenso wichtig wie die quantitativ korrekte Substanzzugabe ist ggf. das effiziente Entfernen der Wirkstoffe nach der Behandlung. Auch dieser „Routineschritt“ ist bei nicht-adhärenten Sphäroiden technisch prekär. Partielle Waschschritte sind manuell möglich, erfordern jedoch zeittechnisch oft die Sammlung und Überführung vieler Kulturen in größere Gefäße und Volumina, ggf. mit nachfolgender Reverteilung in 96-Well-Platten zum weiteren individuellen Monitoring, wobei die ursprüngliche Zuordnung (Sphäroid - Well) allerdings verloren geht. Die mit diesem Vorgang einhergehenden Verluste und Variationen können ebenfalls durch Automatisierung minimiert werden. Unser Pipettierprogramm zur effizienten seriellen Verdünnung des Überstandes beinhaltet zunächst den Well-genauen Transfer der Sphäroide in eine mit frischem Medium bestückte 96-Well-Platte. Für den Transfer stehen Spezialspitzen mit 1,4 Millimeter Öffnung zur Verfügung, die gewährleisten, dass Sphäroide nicht durch Scherkräfte beschädigt werden. Nach Positionierung der Pipettenspitzen und vorsichtiger Sphäroidaspiration in einem größeren Volumen (50 bis 100 Mikroliter pro Well) pausiert der Pipettiervorgang, sodass die Sphäroide zur Spitzenöffnung absinken (Abb. 2). Je nach Sphäroidgröße und -typ sind Zeitintervalle von acht bis zwölf Sekunden erforderlich. Dies erlaubt hernach die akkurate Dispensation der Kulturen in minimalem Flüssigkeitsvolumen (fünf Mikroliter). Mit dieser Vorgehensweise wird eine initiale 1:40-Verdünnung der Wirkstoffe erreicht. Das Programm umfasst zudem zwei intermediäre Verdünnungsschritte $(1: 2,5)$ mit partiellem Austausch des Mediums sowie eine weitere Programmsequenz zum finalen Transfer der Sphäroide auf neu beschichtete Agaroseplatten. Der Zeitaufwand ist zwar erheblich (ca. zehn Minuten für 96 Sphäroide), es kann jedoch standardisiert eine Gesamtverdünnung von 1:10.000 realisiert werden. Da behandelte Sphäroide besonders fragil sind, ist bei Bedarf die Dispensiergeschwindigkeit zu reduzieren. Die Erfolgsrate des automatisierten Prozesses liegt konstant bei über 93 Prozent (Abb. 2), wohingegen die Effizienz bei manuellem Transfer je nach Routine des Experimentators und Bedingung zwischen 50 und 100 Prozent variiert.

Der automatisierte Sphäroidtransfer erlaubt auch eine positionierte Überführung der Sphäroide auf adhärente Oberflächen oder in 3D-ECM-Gele für Migrations- und Invasionsstudien. Erste Ergebnisse implizieren, dass die Automatisierung bessere Voraussetzungen für ein nachfolgendes (Real-Time-)LebendzellImaging schafft. Damit können auch liquid-overlay-Sphäroide diesbezüglich gut genutzt werden, auch wenn die direkte Überschichtung von Sphäroiden mit ECM in ULB-Mikrowellplatten die zunächst einfachere Methodik darstellt [12].

\section{Fazit}

Die Automatisierung von Routinearbeitsschritten in der Multiwell-Sphäroidkultivierung trägt essenziell zur Standardisierung sphäroidbasierter Assays bei und ist im industriellen wie auch akademischen Setting nutzbar. Die parallele Prozessierung großer Mengen individueller Sphäroide verschiedenster GröBenklassen ist auch in liquid overlay standardisiert möglich; methodische und personelle Schwankungen bei der Wirkstoffbehandlung und insbesondere in Langzeitkultur werden minimiert. Die Verknüpfung von teilautomatisierter Multiwell-Sphäroidkultur mit automatisierten Analyseverfahren ist zweckmäBig. 


\section{Literatur}

[1] Kunz-Schughart LA, Kreutz M, Knuechel R (1998) Multicellular spheroids: a three-dimensional in vitro culture system to study tumour biology. Int I Exp Pathol 79:1-23

[2] Kunz-Schughart LA, Freyer JP, Hofstaedter F et al. (2004) The use of 3-D cultures for high-throughput screening: the multicellular spheroid model. J Biomol Screen 9:273-285

[3] Friedrich J, Seidel B, Kunz-Schughart LA et al. (2007) Experimental anti-tumor therapy in 3-D: spheroids-old hat or new challenge? Int J Radiat Biol 83:849-871

[4] Hirschhaeuser F, Menne H, Dittfeld C et al. (2010)

Multicellular tumor spheroids: an under-estimated tool is

catching up again. J Biotechnol 148:3-15

[5] Friedrich J, Seidel B, Ebner R et al. (2009) Spheroid-based drug screen: configurations and practical approach.

Nat Protoc 4:309-324

[6] Hardelauf H, Frimat JP, Stewart ID et al. (2011)

Microarrays for the scalable production of metabolically rele-

vant tumour spheroids: a tool for modulating chemosensitivi-

ty traits. Lab Chip 11:419-428
[7] Friedrich J, Eder W, Castaneda J et al. (2007) A reliable tool to determine cell viability in complex 3-D culture: the acid phosphatase assay. J Biomol Screen 12:925-937

[8] Zhang X, Fryknäs M, Hernlund E et al. (2014) Induction of mitochondrial dysfunction as a strategy for targeting tumour cells in metabolically compromised microenvironments. Nat Commun 5:3295

[9] Ashton TM, Fokas E, Kunz-Schughart LA et al. (2016) The anti-malarial atovaquone increases radiosensitivity by alleviating tumour hypoxia. Nat Commun 7:12308

[10] Ingargiola M, Runge R, Heldt J-M et al. (2014) Potential of a cetuximab-based radioimmuno-therapy combined with external irradiation manifests in a 3-D cell assay. Int J Cancer 135:968-980

[11] Vynnytska-Myronovska B, Bobak Y, Garbe Y et al. (2012) Single amino acid arginine starvation efficiently sensitizes cancer cells to canavanine treatment and irradiation. Int J Cancer 130:2164-2175

[12] Vinci M, Box C, Eccles SA (2015) Three-dimensional (3D) tumor spheroid invasion assay. J Vis Exp 99:e52686

\section{Korrespondenzadresse:}

Prof. Dr. Leoni A. Kunz-Schughart

OncoRay - Nationales Zentrum für Strahlen-

forschung in der Onkologie

Medizinische Fakultät Carl Gustav Carus

Technische Universität Dresden

Fetscherstraße 74

D-01307 Dresden

Tel.: 0351-4587405

leoni.kunz-schughart@oncoray.de

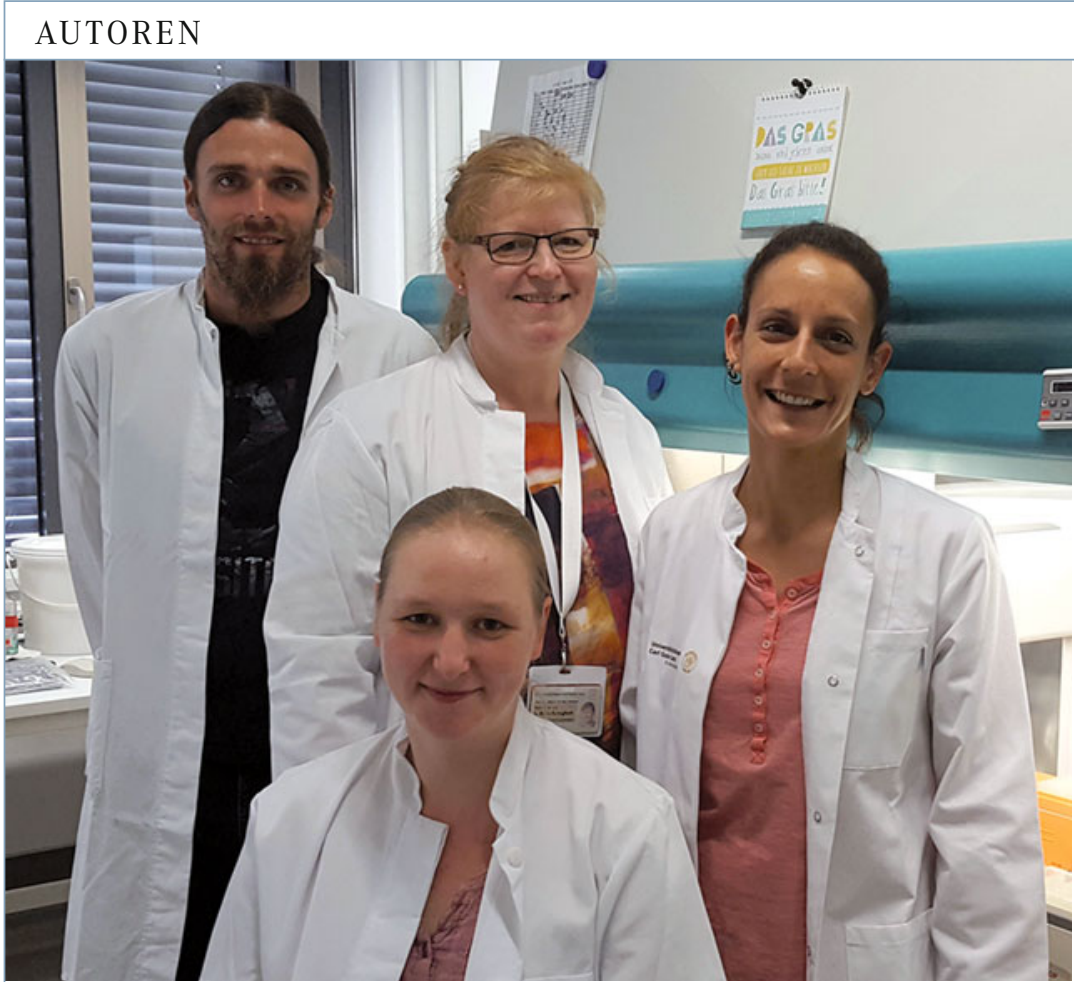

Alexander Krüger, Melanie Hüther, Leoni A. Kunz-Schughart und Mirjam Ingargiola (v. I. n. r.)

\section{Alexander Krüger}

Biologielaborant, 2012-2014 in der AG Tumor-

pathophysiologie am OncoRay, Dresden, verantwortlich für die Entwicklung/Optimierung automatisierter Arbeitsschritte an Sphäroidkulturen, derzeit am Centrum für molekulare Tumordiagnostik, NCT Partnerstandort Dresden.

\section{Melanie Hüther}

MTA, seit 2007 in der AG Tumorpathophysiologie am OncoRay, Dresden, involviert in alle technischen Arbeitsprozesse und Therapiestudien mit Sphäroidkulturen.

\section{Leoni A. Kunz-Schughart}

Seit 2006 Leiterin der AG Tumorpathophysiologie am OncoRay, Dresden. Fokus: Pathophysiologie solider Tumoren, 3DTumormodelle in der kombinatorischen Therapietestung, metabolisches Targeting; frühere Forschungsaktivitäten an den Universitäten Mainz, Regensburg, Oxford, UK, und dem Los Alamos National Laboratory, NM, USA.

\section{Mirjam Ingargiola}

Chemiestudium (Diplom). 2008-2015 als Nachwuchswissenschaftlerin in der AG Tumorpathophysiologie am OncoRay, Dresden, maßgeblich beteiligt an der Etablierung von Langzeitkultur-Sphäroidassays, derzeit Postoc am Institut für Pharmakologie und Toxikologie, TU Dresden. 